\title{
TRAIT-MEDIATED INDIRECT INTERACTIONS IN A SIMPLE AQUATIC FOOD WEB
}

\author{
Scott D. Peacor And Earl E. Werner \\ Department of Biology, University of Michigan, Ann Arbor, Michigan 48109 USA
}

\begin{abstract}
This investigation examines the role of trait-mediated indirect interactions in a simple aquatic food web. We conducted the experiments in cattle watering tanks in order to establish whether competitive and predator-prey interactions between two species are affected by other species in the system; i.e., are pairwise interaction strengths affected by the background species assemblage? We examined the survival and growth response of small bullfrog (Rana catesbeiana) and small green frog (Rana clamitans) tadpoles in the presence and absence of a competitor (large bullfrogs), the lethal presence of the larval odonate predator Tramea lacerata, and the nonlethal (caged) presence of the larval odonate predators Anax junius and Anax longipes. We demonstrate that large bullfrog competitors and caged Anax affect traits (foraging activity level) of small bullfrog and small green frog tadpoles and that these changes in traits, in turn, affect interactions of the small tadpole species with each other and with the other species. In particular, the following four traitmediated indirect interactions were evident: (1) Presence of large bullfrog competitors increased the predation rate of Tramea on small green frogs and small bullfrogs. (2) Presence of nonlethal Anax reduced the predation rate of Tramea on small green frogs. (3) Presence of nonlethal Anax increased the competitive advantage of bullfrogs over green frogs. (4) Presence of nonlethal Anax facilitated midge invasion of the experimental units. The proposed mechanisms (changes in small tadpole activity) involved in these trait-mediated indirect interactions were supported by observational data on tadpole activity and resource levels in the experimental units, and in laboratory experiments examining tadpole activity responses to predators. The occurrence of strong trait-mediated indirect interactions in this simple food web underscores the potential importance of such interactions in animal communities.
\end{abstract}

Key words: Anax; anuran larvae; behavioral induction; Chironomus; higher order interactions; odonate predator; predation risk; Rana; trait-mediated indirect interaction; Tramea.

\section{INTRODUCTION}

It has long been recognized that species in ecological communities interact indirectly with other species through the food web (for reviews see Schoener 1993, Menge 1994, Wootton 1994a). These indirect interactions may take several forms. The most commonly recognized indirect interactions are density-mediated indirect interactions (DMII), or those effects transmitted through changes in densities of intervening species (Abrams et al. 1996). A second form of indirect interaction, termed trait-mediated indirect interactions (TMII; Wootton 1993, Abrams 1995), are those transmitted by induced changes in the traits (behavior, morphology, and life history) of the intervening species. Although we have many examples of DMIIs that clearly have a strong influence on the structure of ecological communities (reviewed in Schoener 1993, Menge 1994, Wootton 1994a), the role of TMIIs has received far less attention and is poorly understood. If traitmediated indirect interactions are quantitatively im-

Manuscript received 6 February 1996; revised 9 August 1996; accepted 22 August 1996; final version received 16 September 1996. portant, however, this will have a large impact on how ecologists conceptualize and model ecological communities (Abrams 1983, Werner 1992a, Wootton 1994b). Consequently, attention to TMIIs and other forms of higher order interactions has increased recently (e.g., Wilbur and Fauth 1990, Werner 1992a, Billick and Case 1994, Wooten 1994b, Abrams et. al 1996).

TMIIs due to behavioral responses are especially likely to be widespread (Werner 1992a). Adaptive (or nonadaptive) behavioral responses to the environment can alter a species' per capita effects on other species and thus lead to TMIIs. For example, many organisms face trade-offs while foraging resulting from the fact that both growth rate and predation risk are positively related to activity levels (reviewed in Lima and Dill 1990, Houston et al. 1993, Werner and Anholt 1993). Werner and Anholt (1993, see also Abrams 1982, 1990, Houston et al. 1993) predict that foraging activity should decrease as roughly the inverse of the square root of the change in mortality risk, and similarly will decrease as resource levels increase. Consequently, both predators and competitors may induce behavioral changes that result in strong TMIIs. Thus the compo- 


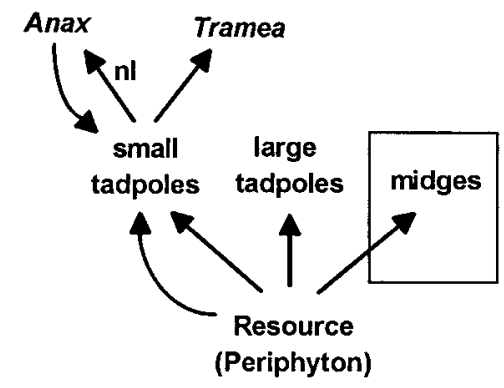

FIG. 1. The experimental food web. Straight arrows represent consumption (density effect) and point in the direction of energy flow; curved arrows represent effects on behavior (trait effect). Anax were isolated in cages and were thus nonlethal (nl). Though not part of the experimental design, midges invaded the experimental units and are therefore in cluded in the diagram. The response of midges (Chironomus spp.) was dependent on both a DMII and a TMII.

nents necessary for strong TMIIs seem plausible, and there are compelling reasons to believe that they will be quantitatively important in many food webs (Werner 1992a, Abrams et al. 1996).

In this study, we test for the occurrence of TMIIs involving responses of small bullfrog (Rana catesbeiana) and green frog (Rana clamitans) tadpoles. Numerous studies have shown that amphibian larvae respond to the above trade-off by reducing activity in the presence of predators (Sih 1987, Lawler 1989, Skelly and Werner 1990, Werner 1991, Semlitsch 1993, Smith and Van Buskirk 1995) and at higher resource levels (Skelly 1995, Werner 1992b, Werner and Anholt 1996). We test whether presence of an odonate predator (Anax junius and Anax longipes) and of a competitor (large bullfrog tadpoles) alter activity levels of small tadpoles, and whether these changes cause TMIIs in this system (Fig. 1). In particular, we tested the following three hypotheses. (1) Competitors (large bullfrogs) reduce resources and thereby induce an increase in foraging activity of the small tadpoles, which, in turn leads to increased predation by the odonate predator Tramea lacerata on the small tadpoles. (2) Predators (Anax) induce a decrease in small tadpole activity which in turn reduces consumption of the small tadpoles by another predator (Tramea) (e. g., Soluk and Collins 1988, Soluk 1993). (3) Predators alter competitive interactions between small tadpole species by differentially affecting activity levels of these species. These hypotheses thus represent TMIIs induced by both predators and competitors. In Hypotheses 1 and 3 the TMIIs have three links, one direct interaction that involves a mediated trait and two direct interactions that involve a change in species density. The terminology of such interaction chains is not well established. For convenience, we will term the indirect interactions TMIIs when at least one link is trait-mediated, and DMIIs when all links are density-mediated.

\section{Methods \\ Cattle tank experiments}

The study was performed in late June 1994 at the University of Michigan's E. S. George Reserve experimental pond site in southern Michigan. The experimental units were polyethylene, cattle-watering tanks $1.9 \mathrm{~m}$ in diameter filled to a water depth of $0.45 \mathrm{~m}$. A cover constructed from $60 \%$ shade cloth prevented oviposition and immigration by aquatic insect predators and competitors. Each tank contained four small, cylindrical predator cages $(11 \mathrm{~cm}$ in diameter and 10.5 $\mathrm{cm}$ long) constructed from slotted plastic drain pipe enclosed by fiberglass window screening. A small piece of polystyrene was placed in each cage to ensure that the cages floated near the surface. Tanks were filled with $1300 \mathrm{~L}$ of untreated well water, and $300 \mathrm{~g}$ of deciduous leaf litter and $25 \mathrm{~g}$ of Purina rabbit chow were added 2 wk before introduction of the tadpoles. Both leaves and rabbit chow are a food source for the tadpoles, and a nutrient source for periphyton. The leaves also provide cover for the tadpoles. One week before introduction of tadpoles, the tanks were inoculated with Daphnia spp. collected from breeding tanks. Daphnia prevent bacterial blooms that reduce oxygen levels and that hinder both periphyton and tadpole growth.

The study system was composed of species found in ponds in Southeastern Michigan. The species with the labile trait necessary for the TMIIs were small, newly hatched bullfrog and green frog larvae (Fig. 1). Final larval instars of the dragonfly Anax were used for the nonlethal predator manipulation, because the presence of Anax has been shown to reduce activity of both small bullfrog and green frog tadpoles (Werner 1991). Final instars of the dragonfly Tramea were used as the lethal predator. Large bullfrog tadpoles, which have exploitative competitive effects on small tadpoles (Werner 1994, Werner and Anholt 1996), served as competitors. Because the large bullfrogs were much larger than the dragonflies, and therefore were not vulnerable to the dragonflies, we assumed that they did not react to the dragonfly's presence (see also Werner and Anholt 1996).

The experiment consisted of a $2 \times 2 \times 2$ factorial design. All eight combinations of 0 or 4 nonlethal (caged) Anax, 0 or 4 lethal (free) Tramea, and 0 or 13 large bullfrog tadpole competitors were included. This density of dragonfly predators represents a low density relative to natural densities of invertebrate predators (McPeek 1990). These treatment combinations were replicated four times (in four spatial blocks). Seventy small bullfrog tadpoles and 70 small green frog tadpoles were added to each tank on 21 June and 22 June, respectively. The small tadpoles were obtained from cultures initiated with several egg masses collected from the E. S. George Reserve experimental ponds (green frogs) or ponds of the Michigan DNR near Sa- 
line, Michigan (bullfrogs). The tadpoles were raised in wading pools filled with well water and fed Purina rabbit chow. Tadpoles in each culture differed in size, and those of intermediate size were selected from each culture. Small green frog tadpoles (average individual wet mass $=13.6 \pm 4.3 \mathrm{mg}$ ) were collected from a single culture initiated with six egg masses. The small bullfrog tadpoles were collected from two separate cultures. Blocks 1 and 3 received tadpoles with an average mass of $19.3 \pm 8.1 \mathrm{mg}$ from one culture initiated with three combined egg masses, while blocks 2 and 4 received slightly smaller tadpoles, $17.1 \pm 6.2 \mathrm{mg}$, from a culture initiated with two combined egg masses. Large bullfrog competitors (average individual mass $=$ $16 \mathrm{~g}$ ) and predators were added June 23. In tanks designated nonlethal predator treatments, 1 Anax was placed in each of the 4 predator cages in that tank. Caged Anax were fed equal numbers of bullfrog and green frog tadpoles ( 4 to 5 of each species for a total mass of $\approx 300 \mathrm{mg}$ ) every other day to ensure a strong predator chemical cue.

During the experiment, the total number of tadpoles visible in the water column or on the tank wall was counted for each tank on four separate days. In addition, the number of tadpoles active was determined (activity defined as those tadpoles either swimming or scraping tank walls). We were unable to distinguish between small bullfrog and green frog tadpoles while conducting these observations.

There was a noticeable difference in periphyton levels between tanks after introduction of tadpoles. We quantified this difference by visually ranking the density of periphyton on the tank walls and floor on a scale of 1 to 5 , where 1 represents a very thin layer of growth (barely perceptible) and 5 represents high density (0.5$1 \mathrm{~cm}$ deep). In addition, during the course of the experiment, larval midges (Chironomus sp.) invaded some of the tanks. Because midges could potentially compete with tadpoles for resources (Morin et al. 1988), we ranked the tank midge invasion on a scale of 1 to $4(1,2,3$, and 4 representing 0,0 to $50 \%, 50 \%$ to $100 \%$, and $\approx 100 \%$ of the leaf and tank surfaces populated, respectively).

The experiment was terminated after $11 \mathrm{~d}$. Small tadpoles were counted and the total wet biomass of each species was determined. We did not include changes in mass of large bullfrog tadpoles as a response variable, because many of the large bullfrogs had begun to metamorphose and therefore lose weight (Werner and Anholt 1996).

\section{Laboratory experiment: effect of predators on tadpole activity}

The large size of the cattle tanks and the shelter provided by the oak leaves made it difficult to make detailed observations on species behavior among treatments. We therefore performed laboratory experiments to further elucidate the mechanisms influencing species interactions. We first tested whether position and activity of green frog tadpoles was influenced by the presence of Anax and Tramea. The experimental unit was a 40-L aquarium filled to within $3 \mathrm{~cm}$ of the aquarium top. Each tank received 10 small green frog tadpoles (average individual mass $=14 \mathrm{mg}$ ). Two clear plastic cups filled with $1 \mathrm{~cm}$ of sand and covered with fiberglass netting held in place by an elastic band were placed next to each other at one end of each tank. Four replicates (spatially blocked) of the following four treatments were established: (1) no predators added to cups, (2) an Anax added to both cups, (3) a Tramea added to both cups, and (4) an Anax added to one cup and a Tramea to the other. Predators, along with 5 small green frog tadpoles as prey, were added to cups $1 \mathrm{~d}$ after the 10 green frog tadpoles were introduced. Observations were initiated $1 \mathrm{~h}$ after predator addition. Three responses were recorded every hour for $7 \mathrm{~h}$ : (1) percentage of tadpoles in the water column or on the tank sides (hereafter termed tadpoles in the water column), (2) percentage of tadpoles moving (scraping the tank bottom or sides, or swimming in the water column), and (3) percentage of tadpoles on the same half of the tank as the predator cups. The following day we again added 5 small green frog tadpoles to each cup containing predators and repeated the observational process every hour for $8 \mathrm{~h}$. All predators consumed all tadpoles fed to them within $2 \mathrm{~h}$ on both days.

\section{Laboratory experiment: effect of Anax presence on} Tramea behavior

A second laboratory experiment was designed to determine whether the presence of Anax affected the behavior of Tramea. The experimental units were $35 \times$ $25 \times 14 \mathrm{~cm}$ plastic containers filled with $7.5 \mathrm{~L}$ of well water. The bottoms of the containers were lined with nylon window screening, and stems of the aquatic plant Myriophyllum sp. were draped lengthwise on one side of the plastic containers to add structure to the experimental units. Two small cylindrical cages $(7.5 \mathrm{~cm} \times$ $2.8 \mathrm{~cm}$ ), constructed of aluminum wire and mesh netting, were placed at both ends of all containers. There were two treatments, a nonlethal Anax and a control treatment, replicated six times. In six containers, one Anax was placed in one of the small cages. One Tramea was then added to each of the twelve containers. After $1 \mathrm{~d}$, the position of the Tramea was recorded every 1.5 $\mathrm{h}$ for $9 \mathrm{~h}$, noting if the Tramea was on or near the vegetation, on the same side of the container as the predator, or on the container bottom. Ideally Tramea's prey should have been present for this test of Anax on Tramea's behavior. However, results of such a test would be confounded by the response of the prey to Anax.

\section{Statistical analysis}

We analyzed the cattle tank experiment results using MANOVA. Survival and average mass gain were the 
dependent variables for small bullfrogs and green frogs. Results were then interpreted using the univariate three-way ANOVA. Because they are not independent within a replicate, responses of the two species were analyzed separately. We also tested whether different species (Tramea, large bullfrogs, and caged Anax) affected small tadpole survival and if these effects were independent. We assumed that if there were no interactions between different species on small tadpole survival that the combined effect of these species would be multiplicative. Thus, in the absence of any interaction, the survival in the presence of two species would be the product of the survival rate in the presence of each species alone. It was thus necessary to log transform the data before analysis in order to test for interactions of different species on small tadpole survival (Billick and Case 1994, Wootton 1994b). We did not test for statistical interactions of factors on mass gain, resource level, and midge density, and thus results were not sensitive to the transformation used. To satisfy data distribution requirements we $\log$ transformed the average mass gain and midge densities. Hypothesis 3 required an analysis of the mass ratios of the two small tadpole species, and this ratio was analyzed in the same manner as the average mass gain.

Cattle tank observational data were averaged over the $4 \mathrm{~d}$ of measurements for each tank. A two-way ANOVA was used to evaluate the effect of competitors and Anax on total number of tadpoles in the water column or on tank sides. Because survival was strongly affected by the lethal presence of Tramea, only data in the absence of Tramea were used. ANOVA was used to evaluate the effect of competitors, Anax, and Tramea on the percentage of visible tadpoles that were active. Mortality due to Tramea does not confound this response. However, in the treatments with both Tramea and Anax, there were many observations in which no tadpoles were visible and thus percentage active was not applicable. Therefore, we did not include the two treatments with both Anax and Tramea in the statistical analysis. An ANCOVA revealed no significant effects of midges on any of the response variables (mass gain, survival, and number of tadpoles in the water column or on tank sides), and the presence of midges was therefore not included in the final analysis of tadpole responses.

The laboratory experiments were analyzed using MANOVA. In both experiments there were three observational responses that were arcsine square-root transformed. For the small green frog behavior experiment, data collected on both days were averaged for each aquarium, and the aquarium means were used in the analysis of variance. Responses were interpreted using univariate ANOVA and Tukey's test for multiple comparisons.

A level of significance of $\alpha=0.05$ was used for all analyses. Lilliefors test and the skewness and kurtosis of the residuals were employed to assure that the an-
TABLE 1. Results of the MANOVA for effects of large bullfrog competitors, caged Anax, Tramea, and blocks on mass gain and survival for small green frogs and small bullfrogs.

\begin{tabular}{lcccl}
\hline \hline \multicolumn{1}{c}{ Source } & df & $\begin{array}{c}\text { Wilks' } \\
\text { lambda }\end{array}$ & $F$ & \multicolumn{1}{c}{$P$} \\
\hline Small green frogs & & & & \\
$\quad$ Large bullfrogs & 2,21 & 0.336 & 20.79 & 0.0005 \\
$\quad$ Caged Anax & 2,21 & 0.503 & 10.39 & 0.001 \\
$\quad$ Tramea & 2,21 & 0.136 & 66.58 & 0.00001 \\
$\quad$ Block & 2,21 & 0.777 & 0.939 & 0.478 \\
Small bullfrogs & & & & \\
$\quad$ Large bullfrogs & 2,21 & 0.336 & 27.22 & 0.0005 \\
$\quad$ Caged Anax & 2,21 & 0.750 & 3.66 & 0.042 \\
$\quad$ Tramea & 2,21 & 0.133 & 71.44 & 0.00001 \\
Block & 2,21 & 0.679 & 1.57 & 0.179 \\
\hline
\end{tabular}

alyzed data did not deviate significantly from normality. In cases where block effects were not significant, designs were collapsed to a complete randomized design.

\section{RESULTS}

The MANOVA on the cattle tank experiment showed that the presence of Tramea, nonlethal Anax, and large bullfrog tadpoles all had significant effects on both small tadpole species (Table 1). Below we interpret the results using the univariate tests.

\section{Average tadpole mass gain}

Presence of free Tramea had no effect on either the average mass gain of small bullfrogs $\left(F_{1,22}=0.95, P\right.$ $=0.34)$ or green frogs $\left(F_{1,22}=0.11, P=0.75\right)$. The presence of caged Anax, however, did have a significant negative effect on green frog average mass gain (Fig. $\left.2, F_{1,22}=11.8, P=0.002\right)$. The overall effect of Anax on small bullfrog tadpole mass gain was not significant $\left(F_{1,22}=3.6, P=0.071\right)$, although this effect was quite clear in the absence of Tramea (Fig. 2). Large bullfrog competitors, in contrast, had a highly significant negative impact on both small bullfrog and green frog average mass gain $\left(F_{1,22}=56.3\right.$ and 38.7 , respectively, $P<0.0001$ for both). Small bullfrog tadpoles in blocks 1 and 3 were $\approx 1.35$ times larger than in blocks 2 and 4 (block effect; $F_{3,22}=3.3, P=0.039$ ). There was no effect of block on small green frog mass gain $\left(F_{3,22}=\right.$ $1.5, P=0.25)$.

\section{Number tadpoles surviving}

Mortality of free Tramea was minimal. In three tanks one dead Tramea was recovered, and in one tank one was missing. The number of small bullfrog and green frog tadpoles surviving was reduced by approximately half in the presence of these free Tramea $\left(F_{1,22}=122\right.$ and 136, respectively, $P<0.0001$ for both; Fig. 3). The presence of competitors also reduced small bullfrog and green frog survival $\left(F_{1,22}=11.5, P=0.003\right.$ and $F_{1,22}=11.0, P=0.003$, respectively), but this effect was only evident in the presence of Tramea (Fig. 


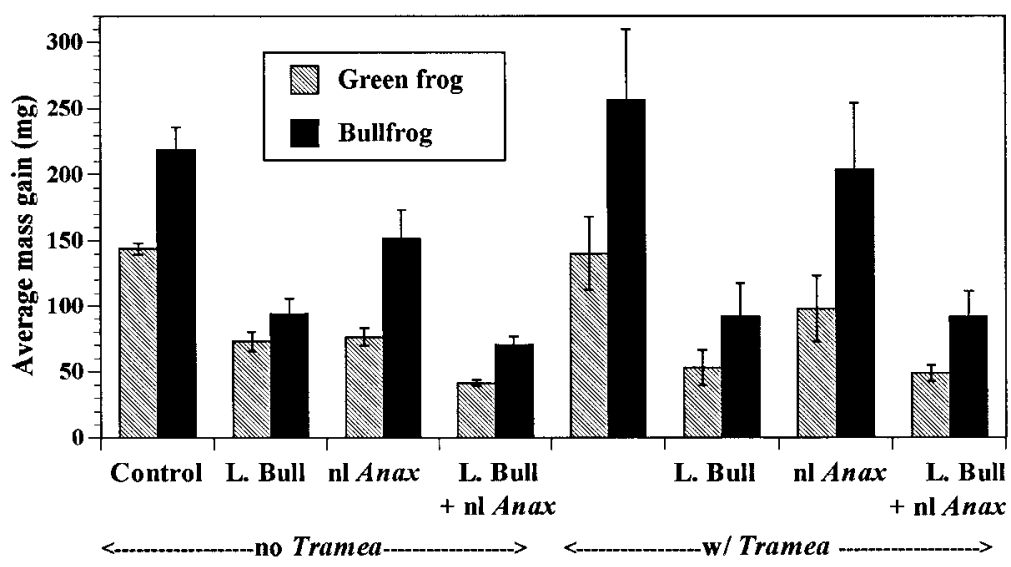

FIG. 2. The average mass gain of small green frog and small bullfrog tadpoles in all eight treatments. Error bars represent the standard error, $\mathrm{nl}=$ nonlethal, $\mathrm{L}$. Bull = large bullfrog tadpoles.

3). The nonlethal presence of Anax had a significant positive effect on green frog survival $\left(F_{1,22}=6.3, P\right.$ $=0.02)$, but not bullfrog survival $\left(F_{1,22}=1.5, P=\right.$ 0.216). The effect of Anax on green frog survival came primarily in the presence of Tramea. There was a significant Tramea by competitor interaction term for both small bullfrog $\left(F_{1,22}=10.6, P=0.003\right)$ and green frog tadpoles $\left(F_{1,22}=5.3, P=0.031\right)$. The Tramea by Anax interaction was marginally significant $\left(F_{1,22}=4.2, P\right.$ $=0.052)$ for green frogs but not significant for bullfrogs $\left(F_{1,22}=0.54, P=0.45\right)$. Survival of large bullfrog tadpoles was $100 \%$.

\section{Resource levels}

Large bullfrogs significantly reduced resource levels $\left(F_{1,32}=20.4, P<0.0001\right)$. Fifteen of the 16 tanks with large bullfrogs received low rankings (1 and 2) of periphyton levels, while only two treatments without large bullfrogs had low periphyton levels. All 12 tanks with high periphyton levels (rank 4 and 5) did not have large bullfrogs. The nonlethal presence of Anax led to an increase in resource levels $\left(F_{1,32}=4.65, P=0.04\right)$. There was no observable effect of Tramea on the resource levels $\left(F_{1,32}=0.002, P=0.96\right)$.

\section{Midge density}

There was a strong effect of treatment on midge density (Fig. 4). The 16 tanks with large bullfrogs had no visible sign of midges (rank 1). There was a significant effect of Tramea $\left(F_{1,14}=1.18, P=0.002\right)$ and Anax $\left(F_{1,14}=1.22, P=0.002\right)$ on midge density. All tanks with predators were invaded by midges in the absence of large bullfrogs (rank 3 and 4). In contrast, in the treatment without predators and without large bullfrogs midge invasion was very low (in 3 tanks there was no sign of midges [rank 1] and in one tank there was a very low density [rank 2]). Thus the presence of predators, both nonlethal Anax and lethal Tramea, modified the tank environment in a way that facilitated invasion by midges, but only in the absence of large bullfrogs.

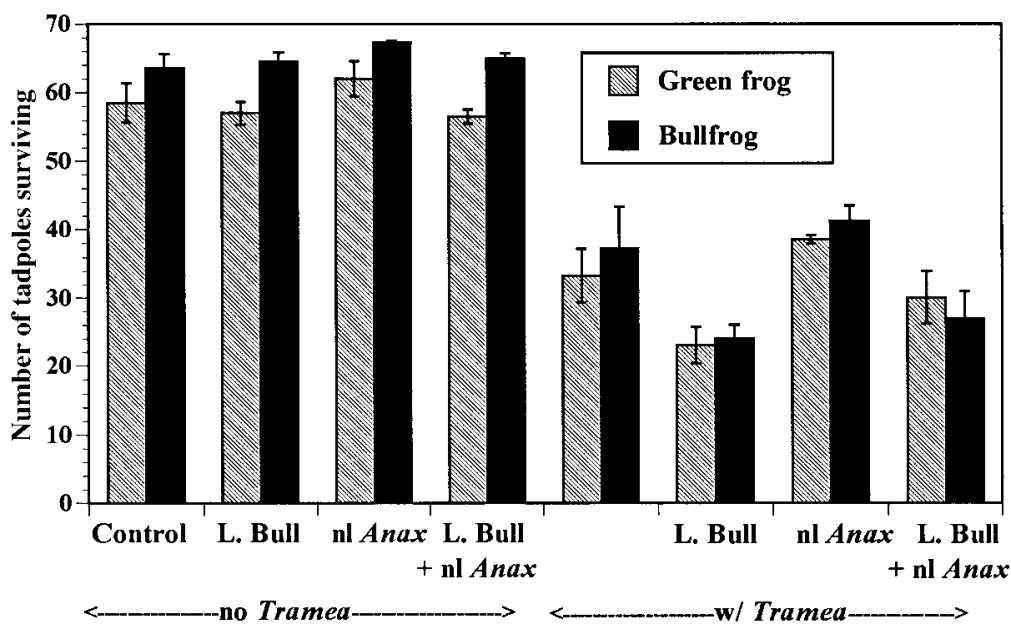

FIG. 3. The number of surviving small green frog and small bullfrog tadpoles, from an initial 70, in all eight treatments. Error bars represent the standard error, $\mathrm{nl}=$ nonlethal, L. Bull = large bullfrog tadpoles. 


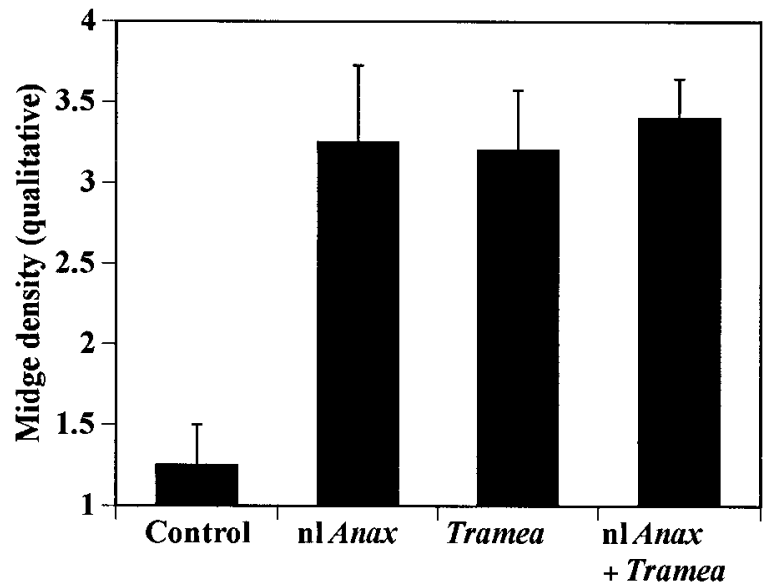

FIG. 4. Midge density rank. There were no midges in any tanks with large bullfrogs (not shown). Midge invasion was almost exclusively restricted to tanks without large bullfrogs and with either nonlethal or lethal predators.

\section{Tadpole behavior in the cattle tank experiment}

Small tadpole behavior was highly responsive to the presence of predators and competitors (Fig. 5). Fewer tadpoles in the cattle tanks were observed in the water column or on the tank sides in treatments with Anax or large bullfrogs (Fig. 5a; $F_{1,12}=47.4, P=0.00002$ and $F_{1,12}=12.0, P=0.0047$, respectively). Although the number of tadpoles in the water column was negatively correlated with the presence of both predators and competitors, competitors and predators had opposite effects on the tadpole's activity. A significantly greater fraction of those tadpoles in the water column or on the tanks sides were active when large bullfrogs were present (Fig. 5b, $F_{1,19}=10.9, P=0.0038$ ), whereas a significantly smaller fraction were active in the presence of caged Anax (Fig. 5b, $F_{1,19}=18.5, P=$ 0.00038). Although Tramea did not have a significant effect on the number of tadpoles active $\left(F_{1,19}=2.8, P\right.$ $=0.11)$, there was a trend toward fewer tadpoles active in their presence (Fig. 5b). Recall that the caged Anaxfree Tramea and caged Anax-free Tramea-large bullfrog treatments were not included in the statistical analysis of the number active, because so few were seen in the water column or on the tank sides. In these two treatments, a total of 18 and 3 tadpoles, respectively, were observed in the water column for all 16 observations ( 4 replicates $\times 4 \mathrm{~d}$ ), and none of them were active.

\section{Laboratory experiments}

Changes in tadpole behavior in the presence of odonate larvae in the laboratory experiment were consis-

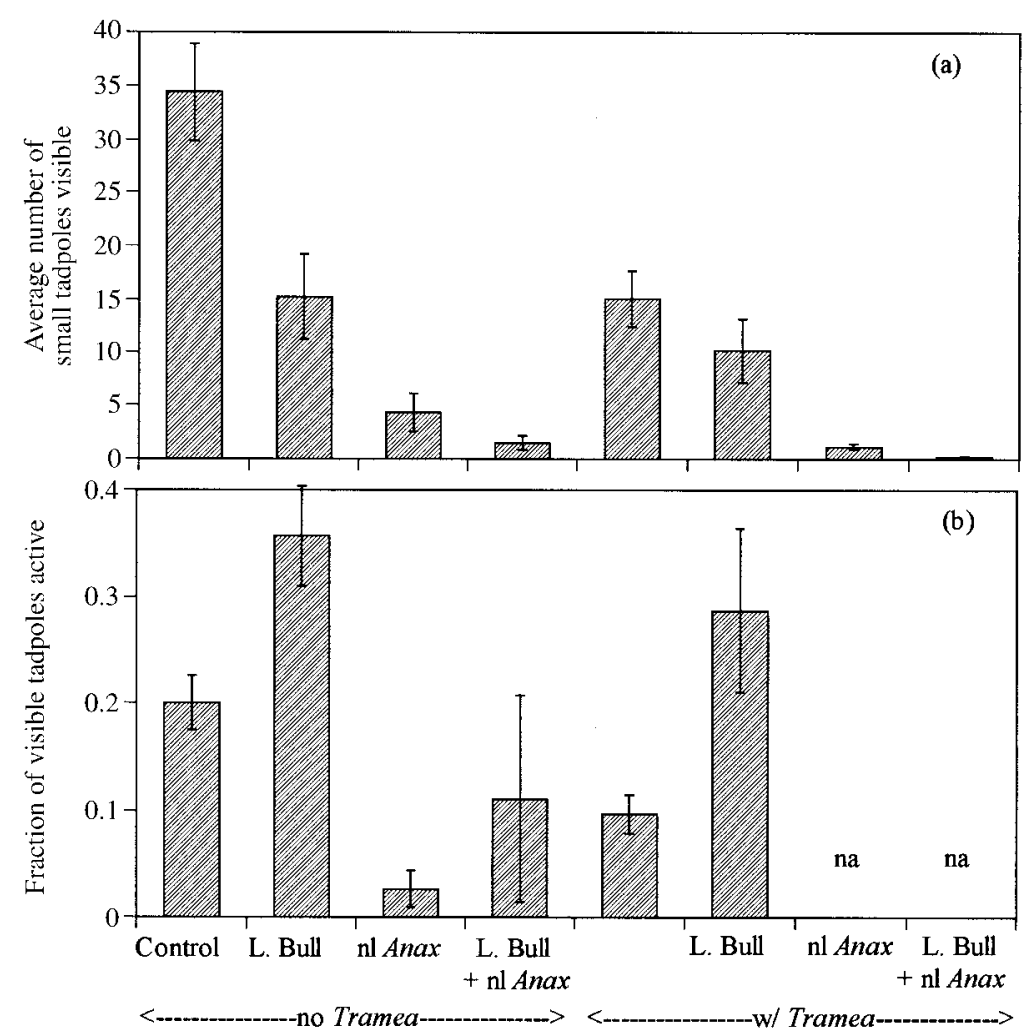

FIG. 5. (a) The average number of small tadpoles visible: i.e. those on the tank walls or in the water column. Tramea, Anax, and large bullfrogs all have a significant effect on the position of the tadpoles. (b) The percentage of visible tadpoles that were active. In two treatments there were too few tadpoles visible to quantify this response, indicated by na (not applicable). 


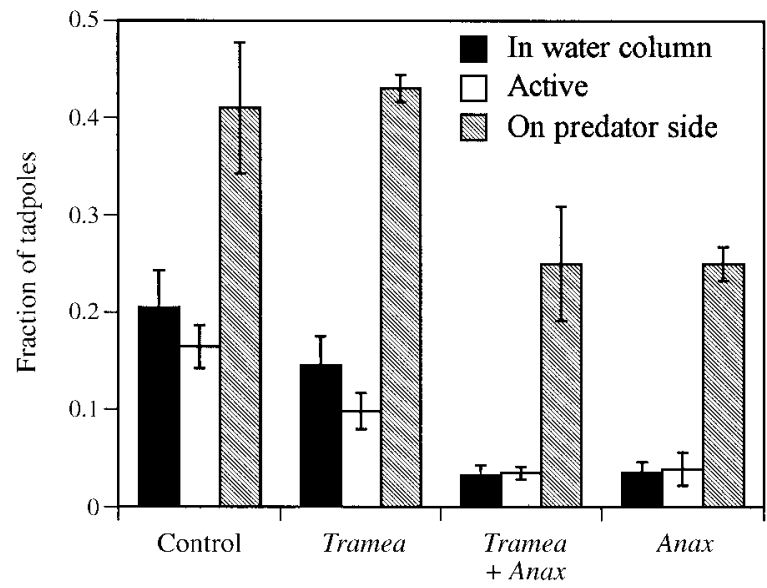

FIG. 6. Effect of Tramea and Anax on small green frog tadpole behavior. Fraction of tadpoles in the water column (= not on the aquarium floor), fraction of tadpoles active, and the fraction of tadpoles on the predator cage side of the aquarium are all indicated. Observations were averaged for each tank. Error bars are the standard errors of the treatment averages determined from these values. The presence of two Anax and of one Anax and one Tramea both had significant effects on small green frog tadpole activity and spatial distribution.

tent with the cattle tank observations. Caged dragonflies had a significant effect on the behavior of green frog tadpoles in the aquaria (Fig. 6; MANOVA, $F_{9,24}$ $=3.5, P=0.007)$. The univariate tests showed a significant treatment effect on both activity level and position of green frog tadpoles (Fig. 6; percentage active $F_{3,12}=12.27, P=0.0006$; percentage in water column $F_{3,12}=15.58, P=0.0002$; percentage on predator side $\left.F_{3,12}=4.37, P=0.026\right)$. Tukey's test for multiple comparisons showed that, in the presence of Anax, tadpoles reduced their activity and presence in the water column, and their spatial distribution was biased away from the predator's neighborhood. For example, in the control $21 \%$ of the tadpoles were in the water column and $16 \%$ were active, whereas only $3.5 \%$ were in the water column and $4 \%$ were active if two nonlethal Anax were present (Fig. 6). This represents a significant decrease for both responses $(P=0.0014$ and 0.0010 for percentage in water column and percentage active, respectively). In contrast, the presence of Tramea did not have a significant effect on the activity level or the position of the green frog tadpoles relative to the control (Fig. 6; $P=0.14,0.48$, and 0.99 , for percentage active, percentage in the water column, and percentage on side opposite the predator cage, respectively), but these responses were significantly different from both treatments with Anax. The reaction of the tadpoles to the treatment with two Anax and the treatment with one Anax and one Tramea was practically identical for all three responses, indicating that the presence of one Anax in an aquarium was enough to exceed a response threshold.
Anax did not affect the position of Tramea in the Anax-Tramea laboratory experiment. Although Tramea frequently moved, there was no difference in Tramea position between Anax treatments and control treatments $\left(F_{1,10}=1.6, P=0.25\right)$.

\section{Discussion}

The results of the cattle tank experiment confirm the three predicted trait-mediated indirect interactions (TMII). In addition, a TMII strongly affected invasion of the tanks by midges. We discuss each TMII separately and then compare them with results in the literature.

\section{The non-lethal effect of Anax on the per capita predation rate of Tramea on small tadpoles}

We hypothesized that the presence of Anax would decrease foraging activity of small tadpoles, which in turn would decrease predation by Tramea on the small tadpoles. The nearly significant interaction term $(P=$ 0.052) between presence of Anax and Tramea on survival of green frogs supports this hypothesis. Although the interaction term is marginal, it is well recognized that the ANOVA resolves main effects more efficiently than interactions (Wade 1992). Interaction effects may contribute to significant statistical main effects while remaining insignificant themselves (see Wade 1992). Our results appear to be an example. The significant main effect of nonlethal Anax on survival is due almost entirely to differences in the presence of Tramea. This is verified by the application of a two-way ANOVA to treatments either with or without Tramea. In the presence of Tramea, the addition of Anax has a significant positive effect on green frog survival $\left(F_{1,13}=5.7, P\right.$ $=0.033)$, while in the absence of Tramea there is no significant effect of nonlethal Anax $\left(F_{1,13}=0.49, P=\right.$ 0.50 , see Fig. 3). Our data thus support a positive TMII of Anax on the survival of green frog tadpoles.

The observational data from the cattle tank and laboratory experiments support the above interpretation. Nonlethal Anax had a strong effect on the activity level of tadpoles; those tadpoles in the water column or tank sides were much less active in the presence of Anax (Fig. 5). The laboratory experiment also demonstrated that green frog activity was greatly reduced if Anax was present (Fig. 6). We suggest that the decrease in tadpole activity induced by presence of nonlethal Anax led to the decrease in predation rates by Tramea.

It is also possible that there are counteracting effects occurring in our design. Anax, in addition to inducing a decrease in tadpole activity, caused the tadpoles to spend more time on the tank bottoms, where Tramea are located, and this may increase predation on tadpoles. The Anax-induced reduction in mass gain could also lead to increased predation by Tramea as smaller individuals may be more vulnerable. A more sophisticated design is required to evaluate the relative contributions of these possible counteracting effects. 


\section{Large bullfrog effect on the per capita predation rate of Tramea on small tadpoles}

Presence of large bullfrog tadpoles increased the predation rates of Tramea on small bullfrog and green frog tadpoles. The mechanism most likely responsible for this effect is that large bullfrogs, by reducing resources, caused the target species to forage more actively and thereby become more vulnerable to predators. This mechanism was supported by observational data; there was a clear effect of large bullfrogs on resource levels, and those small tadpoles visible in the water column or on the tanks sides were approximately twice as active in the presence of large bullfrogs as in their absence. Further, Anholt and Werner (1995) demonstrated that small bullfrog tadpoles are more active at artificially manipulated lower food levels, and that at these higher activity levels predation by Tramea was greater.

Though we believe that the reduction in resources due to induced behavioral changes is the major effect responsible for the competitor induced TMII, there are several alternative hypotheses. First, small tadpoles swim away from the larger tadpoles when they are disturbed by the larger tadpoles movement (S. D. Peacor and E. E. Werner, personal observation). This represents an increase in small tadpole movement, and could increase vulnerability to predators. Second, the reduction in resources by the large bullfrogs led to a reduction in the mass gain of the small tadpoles (Fig. 2 ). This could also indirectly affect their survival, as smaller tadpoles may be more susceptible to Tramea predation. We note, however, that the caged Anax also led to a very significant decrease in mass gain (Fig. 2), but their effect on tadpole mortality by Tramea was opposite that of large bullfrogs. Predation by Tramea on small green frogs was lower when Anax was present, and the trend for the small bullfrogs was in the same direction (Fig. 3). Most likely the changes in both tadpole growth rate and tadpole activity level caused by large bullfrogs and Anax influenced the predation rate of Tramea on small tadpoles. Determination of the relative magnitudes of these effects would require a more detailed examination of Tramea predation rates on small tadpoles as a function of size and activity. Contrasting treatment effects, however, make it clear that induced changes in activity levels are affecting the survivorship of small tadpoles.

\section{Nonlethal effect of Anax on the competitive interaction between small green frogs and small bullfrogs}

The relative mass gains of small bullfrog and green frog tadpoles also depended on treatment and support Hypothesis 3. In Fig. 7, the ratio of small bullfrog to small green frog tadpole mass gain is illustrated for treatments without Tramea (i.e., treatments in which survivorship of tadpoles was relatively constant). The mass gain of small bullfrogs was higher than that of

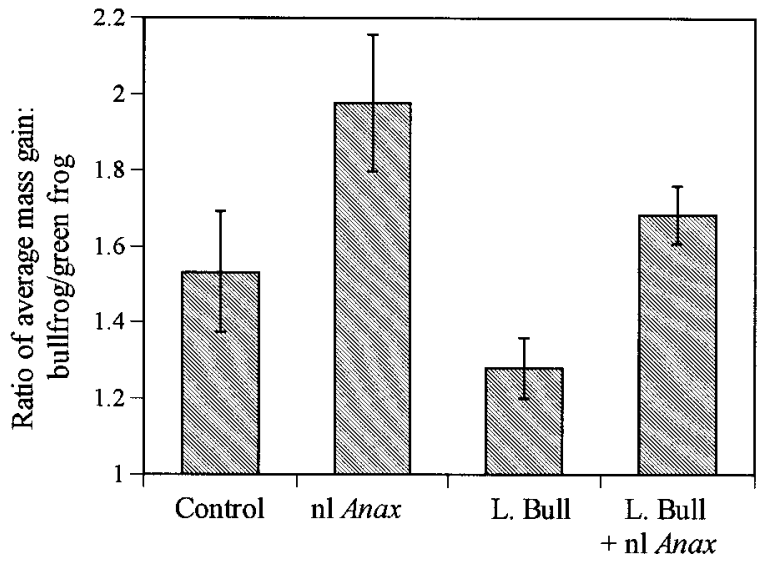

FIG. 7. The ratio of the average mass gain of small bullfrog tadpoles over small green frog tadpoles. The relative mass gain of small bullfrog tadpoles was greater when nonlethal (caged) Anax were present.

green frogs for all treatments. This is likely due to their higher activity levels (Werner 1991). What is also evident, however, is that when nonlethal Anax were present, the small bullfrogs advantage in mass gain was highest (Fig. 2; ANOVA, $F_{1,12}=7.5, P=0.02$ ). Small tadpole growth rate should accelerate as the tadpoles get larger (Werner 1994), and we thus might expect the relative mass gain of small bullfrogs to be largest in treatments where they have the highest mass gain, or those treatments without nonlethal Anax. Thus, the nonlethal presence of a predator affects the competitive interaction between these two frog species. This result is consistent with an earlier laboratory experiment, and it appears that the mechanism responsible is that the proportional decrease in activity is greater for the green frog than the bullfrog in the presence of Anax (Werner 1991).

\section{TMII between Anax and midges}

The strong treatment effects on midge density suggests the existence of a fourth TMII in our experiment. Adult midges apparently penetrated the shade cloth covers and reproduced. We quantified their presence, because we expected that they could have competitive effects on the tadpoles (Morin et al. 1988). We did not expect a TMII from the predator on midge abundance, but this became evident on analysis of the data.

The data suggest that the impact of tadpole foraging on resource levels determined midge success. In treatments with large bullfrogs there was no invasion by midges. This is likely due to an indirect effect of large bullfrogs on midges via a reduction in resource levels, as resource levels were significantly reduced by large bullfrogs. In tanks lacking large bullfrogs, midges were almost totally absent in the no predator treatments but successfully invaded all tanks with predators. Both predators likely interacted indirectly with the midges via their influence on small tadpoles (Fig. 1). Appar- 
ently, in the absence of nonlethal Anax and Tramea, the small tadpoles reduced resources to a level that prohibited midge invasion. With predators, however, resources were released and midges were able to colonize the system. Anax significantly increased resource levels by altering the foraging activity of the tadpoles. Although Tramea reduced tadpole density by $\approx 50 \%$, we did not observe significant changes in resource level. We suspect that a more rigorous, quantitative test of resource levels would indicate that Tramea, like Anax, had a positive affect on resource levels. Our data thus supports the existence of a TMII between Anax and midges, and are suggestive of a DMII between Tramea and midges. Alternatively, it is possible that tadpole foraging activity interfered with establishment of early instars of the midges, and that this was the mechanism of the TMII and the DMII.

\section{Nonlethal effects of Tramea}

In contrast to the behavioral changes of tadpoles in the presence of Anax, the presence of Tramea did not invoke a significant change in tadpole activity level or tadpole position in either the cattle tank or laboratory experiments. However, probability values for both experiments were quite low, and the data suggest a similar, but much weaker trend, than that with Anax (Fig. $5 b$ and 6). The dramatic differences in tadpole behavioral responses to Anax and Tramea is a surprising result given that these are both voracious odonate predators. These results underscore the sensitivity of behavioral reactions of prey to different predators.

We also found that Tramea do not appear to be affected by the presence of Anax. Because there are small effects on or from Tramea, such effects do not confound our interpretation of the small tadpole survival results.

\section{General discussion}

Our results reinforce those of other studies demonstrating predator-induced TMIIs and additionally illustrate a competitor-induced TMII. First, the presence of a predator may induce a change in habitat use or foraging activity of a second predator, which in turn lowers predation rates on a consumer at the bottom of a three level trophic chain (Carpenter et al. 1987, Turner and Mittelbach 1990) or on a shared prey (Soluk and Collins 1988, Huang and Sih 1990, Soluk 1993, Wissinger and McGrady 1993). In these examples, the effect of the predator on its prey modifies the prey's predation rate on its resource. The activity change may also affect the prey's vulnerability to other predators. Soluk and Collins (1988) and Soluk (1993) show that in a two-level system with two predators and one prey, the presence of one predator can change prey behavior such that the predation rate by the second predator changes. Our study also demonstrates this TMII: the nonlethal presence of Anax changed the behavior of small green frog tadpoles, which in turn made them less vulnerable to Tramea. A predator also may induce behavioral changes in competitors leading to differential changes in consumption rates of a shared resource, thus altering competitive interactions (Werner et al. 1983, Werner 1991, Werner and Anholt 1996). This investigation also demonstrated this TMII (Hypothesis 3 and midge invasion success). Other investigations, although they have not demonstrated changes in responses such as growth rate or survival, strongly suggest a similar change in competitive interactions due to the presence of a predator (Feener 1981, Kohler and McPeek 1989, Bouskila 1995).

We have also demonstrated that a competitor may be responsible for TMIIs. Large bullfrogs, by inducing behavioral changes in small tadpoles via resource depletion, led to increased mortality of the small tadpoles by Tramea. Further, in our system, the changes due to competitors led to a stronger TMII than did the presence of another predator. This interaction differs from the above examples in that the cause of the trait change is not due to predation risk, but rather change in resource levels. Anholt and Werner (1995) also have demonstrated that predation rates on a target species depends on the target species' resource levels by directly manipulating resource levels.

Both types of TMIIs, those caused by changes in predation risk and those caused by changes in resource levels via competition, may be quite general. In order to maximize fitness, many species must balance predation risk and growth rate by adjusting activity level (Abrams 1984, 1990, Houston et al. 1993, Werner and Anholt 1993). Changes in both perceived predation risk and resource level will shift optimal activity level, which in turn may lead to a change in the strength of competitive or predatory interactions with other species in the community-all examples of TMII.

This investigation contributes to a growing realization that TMIIs are likely common in nature by demonstrating four TMIIs in the simple foodweb in Fig. 1. It is clear that these TMIIs can have strong quantitative effects on components of species' fitness (growth, survival) in short-term experiments. Theory casting these effects in a population dynamic framework suggests that these effects may have non-intuitive consequences to community structure (e.g., Abrams 1984, 1995, Matsuda et al. 1995). The empirical questions that need to be addressed at this point concern the role these TMIIs play in community structure. Will they affect the species that coexist in a community? How widespread are TMIIs in different systems, environments, and taxa? If species interactions are dependent on the composition of communities, does this lend stability or instability to these systems? How important are TMIIs as a selective force in shaping the characteristics of species? If TMIIs have as strong an effect on community structure as their short-term effect on components of fitness would suggest, then, at least in some systems, we will 
need to rethink the way that we conceptualize species interactions in community theory.

\section{ACKNOWLEDGMENTS}

We wish to thank Rick Relyea for assistance in conducting the experiments. We also thank James Gapzynski and Allan Sutton of the Michigan Department of Natural Resources for permission to collect, and help in collecting, bullfrog eggs from the Saline Pond Facility. Comments from Peter Abrams, Arthur Benke, Peter Eklöv, Rick Relyea, and an anonymous reviewer greatly improved the manuscript. This work was supported by NSF grant DEB-9119948 to Brad Anholt and Earl Werner.

\section{Literature Cited}

Abrams, P. A. 1982. Functional responses of optimal foragers. American Naturalist 120:382-390.

1983. Arguments in favor of higher-order interactions. American Naturalist 121:887-891.

- 1984. Foraging time optimization interactions in food webs. American Naturalist 124:80-96.

1990. The effects of adapative behavior on the type-2 functional response. Ecology 71:877-885.

. 1995. Implications of dynamically variable traits for identifying, classifying and measuring direct and indirect effects in ecological communities. American Naturalist 146: $112-134$.

Abrams, P. A., B. A. Menge, G. G. Mittelbach, D. Spiller, and P. Yodzis. 1996. The role of indirect effects in food webs. Pages 371-395 in G. Polis and K. Winemiller, editors. Food webs: dynamics and structure. Chapman and Hall, New York, New York, USA.

Anholt, B. R., and E. E. Werner, 1995. Interaction between food availability and predation mortality mediated by adaptive behavior. Ecology 76:2230-2234.

Billick, I., and T. J. Case. 1994. Higher order interactions in ecological communities: what are they and how can they be detected? Ecology 75:1529-1543.

Bouskila, A. 1995. Interactions between predation risk and competition: a field study of kangaroo rats and snakes. Ecology 76:165-178.

Carpenter, S. R., J. F. Kitchell, J. R. Hodgson, P. A. Cochran, J. J. Elser, M. M. Elser, D. M. Lodge, C. Kretchmer, and X. He. 1987. Regulation of lake primary productivity by food web structure. Ecology 68:1863-1876.

Feener, D. H. 1981. Competition between ant species: outcome controlled by parasitic flies. Science 214:815-817.

Houston, A. I., J. M. McNamara, and J. M. C. Hutchinson. 1993. General results concerning the trade-off between gaining energy and avoiding predators. Philosophical Transactions Royal Society London 341:375-397.

Huang, C., and A. Sih. 1990. Experimental studies on behaviorally mediated, indirect interactions through a shared predator. Ecology 71:1515-1522.

Kohler, S. L., and M. A. McPeek. 1989. Predation risk and the foraging behavior of competing stream insects. Ecology 70:1811-1825

Lawler, S. P. 1989. Behavioral responses to predators and predation risk in four species of larval anurans. Animal Behaviour 38:1039-1047.

Lima, S. L., and L. M. Dill. 1990. Behavioral decisions made under the risk of predation: a review and prospectus. $\mathrm{Ca}$ nadian Journal of Zoology 68:619-640.

Matsuda, H., P. A. Abrams, and M. Hori. 1995. The effect of adaptive anti-predator behavior on exploitative competition and mutualism between predators. Oikos 68:549559.
McPeek, M. A. 1990. Determination of species composition in the Enallagma damselfly assemblages of permanent lakes. Ecology 71:83-98.

Menge, B. A. 1994. Indirect effects in marine rocky intertidal interaction webs: pattern and importance. Ecological Monographs 65:21-74.

Morin, P. J., S. P. Lawler, and E. A. Johnson. 1988. Competition between aquatic insects and vertebrates: interaction strength and higher-order interactions. Ecology 69:14011409.

Schoener, T. W. 1993. On the relative importance of direct versus indirect effects in ecological communities. Pages 365-411 in H Kawanabe, J. E. Cohen, and K. Iwasaki, editors. Mutualisms and community organization. Oxford University Press, Oxford, England.

Semlitsch, R. D. 1993. Effects of different predators on the survival and development of tadpoles from the hybridongenetic Rana esculenta complex. Oikos 67:40-46.

Sih, A. 1987. Predators and prey lifestyles: An evolutionary and ecological overview. Pages 203-224 in W. C. Kerfoot and A. Sih, editors. Predation: direct and indirect impacts on aquatic communities. University of New England Press, Hanover, New Hampshire, USA.

Skelly, D. K. 1995. A behavioral tradeoff and its consequences for the distribution of Pseudacris treefrog larvae. Ecology 76:150-164.

Skelly, D. K., and E. E. Werner. 1990. Behavioral and lifehistorical responses of larval American toads to an odonate predator. Ecology 71:2313-2322.

Smith, D. C., and J. Van Buskirk. 1995. Phenotypic design, plasticity, and ecological performance in two tadpole species. American Naturalist 145:211-233.

Soluk, D. A. 1993. Multiple predator effects: Predicting combined functional response of stream fish and invertebrate predators. Ecology 74:219-225.

Soluk, D. A., and N. C. Collins. 1988. Synergistic interactions between fish and stoneflies: facilitation and interference among stream predators. Oikos 52:94-100.

Turner, A. T., and G. G. Mittelbach. 1990. Predation avoidance and community structure: interactions among piscivores, planktivores and plankton. Ecology 71:2241-2254.

Wade, J. W. 1992. Sewall Wright: gene interaction and the Shifting Balance Theory. Pages 35-62 in J. Antonovics and D. Futuyma, editors. Oxford surveys of Evolutionary Biology VI. Oxford University Press, Oxford, England.

Werner, E. E. 1991. Nonlethal effects of a predator on competitive interactions between two anuran larvae. Ecology 72:1709-1720.

$1992 a$ Individual behavior and higher-order species interactions. American Naturalist 140:s5-s32.

$1992 b$ Competitive interactions between wood frog and northern leopard frog larvae: the influence of size and activity. Copeia 1992:26-35.

-1994. Ontogenetic scaling of competitive relations: size-dependent effects and responses in two anuran larvae. Ecology 75:197-213.

Werner, E. E., and B. R. Anholt. 1993. Ecological consequences of the trade-off between growth and mortality rates mediated by foraging activity. American Naturalist 142: 242-272.

Werner, E. E., and B. R. Anholt. 1996. Predator-induced behavioral indirect effects: Consequences to competitive interactions in anuran larvae. Ecology 77:157-169.

Werner, E. E., J. F. Gilliam, D. J. Hall, and G. G. Mittelbach. 1983. An experimental test of the effects of predation risk on habitat use in fish. Ecology 64:1540-1548.

Wilbur, H. M., and J. E. Fauth. 1990. Experimental aquatic foodwebs: interactions between two predators and two prey. American Naturalist 135:176-204. 
Wissinger, S., and J. McGrady. 1993. Intraguild predation and competition between larval dragonflies: direct and indirect effects of shared prey. Ecology 74:207-218.

Wootton, J. T. 1993. Indirect effects and habitat use in an intertidal community: interaction chains and interaction modifications. American Naturalist 141:71-98. 1994a. The nature and consequences of indirect effects in ecological communities. Annual Review of Ecology and Systematics 25:443-466.

1994b. Putting the pieces together: testing the independence of interactions among organisms. Ecology 75: $1544-1551$ 